6 - ORIGINAL ARTICLE

EXPERIMENTAL NEUROLOGY

\title{
Evaluation of nerve regeneration in diabetic rats $^{1}$
}

\author{
Guataçara Schenfelder Salles JrI, José Carlos Marques de Faria ${ }^{\mathrm{II}}$, Fábio Freitas Busnardo ${ }^{\mathrm{III}}$, Rolf Gemperli ${ }^{\mathrm{IV}}$, Marcus Castro \\ Ferreira $^{\mathrm{v}}$ \\ IFellow PhD degree, Postgraduate Program in Clinical Surgery, Division of Plastic Surgery, Department of Surgery, School of Medicine, University \\ of Sao Paulo (FMUSP), Brazil. Conception, design and scientific content of the study; technical procedures; analysis and interpretation of data; \\ manuscript writing. \\ IIPhD, Surgeon, Division of Plastic Surgery, Department of Surgery, Clinics Hospital, FMUSP, Sao Paulo-SP, Brazil. Intellectual and scientific content \\ of the study, interpretation of data, manuscript writing, critical revision. \\ IIIPhD, Surgeon, Division of Plastic Surgery, Department of Surgery, Clinics Hospital, FMUSP, Sao Paulo-SP, Brazil. Critical revision.

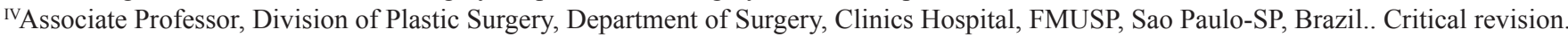 \\ ${ }^{\vee}$ Full Professor, Head, Division of Plastic Surgery, Department of Surgery, Clinics Hospital, FMUSP, Sao Paulo-SP, Brazil. Critical revision.
}

\section{ABSTRACT}

PURPOSE: To compare sciatic nerve regeneration between non-diabetic (control) and streptozotocin-induced diabetic Wistar rats.

METHODS: Four subgroups were evaluated. CN: Non-diabetic rats submitted to neurorrhaphy ( $\mathrm{n}=9$ ); DN: Diabetic rats submitted to neurorrhaphy $(n=9)$; CG: Non-diabetic rats submitted to nerve grafting $(n=10)$; DG: Diabetic rats submitted to nerve grafting ( $n=9)$. The nerve regeneration was evaluated by walking track analysis (sciatic functional index), electrophysiological test, histomorphometric analysis and triceps surae muscle weight.

RESULTS: At 60 days post-surgery, functional recovery of DN was similar to that of the non-diabetic rats (CN, CG), but DG didn't achieve the same. Evoked potential amplitudes showed no statistically significant differences among subgroups. Triceps surae muscle was heavier in $\mathrm{CN}$. No statistically significant differences were observed between the control and diabetes subgroups with respect to histomorphometric analysis.

CONCLUSION: After 60 days, DN had a functionally similar recovery to that of the control animals, whereas nerve grafting in diabetic rats didn't allow the same. The muscle atrophy was lower in CN. In the rest of evaluations, as electrophysiological and histomorphometric, diabetic rats were not different from control ones.

Key words: Diabetes Mellitus, Experimental. Sciatic Nerve. Transplants. Nerve Regeneration. Rats. 


\section{Introduction}

Diabetes mellitus (DM) is an increasingly important issue in public health worldwide, resulting in the loss of productivity, lower quality of life and decreased life expectancy. The overall number of individuals with DM has increased from 153 million in the 1980 s to 347 million in recent years ${ }^{1}$.

Some authors believe that there is impaired nerve regeneration in DM because there may be microangiopathic involvement of the vasa nervorum (the vascular supply of the peripheral nerves), resulting in an unfavorable environment for nerve regeneration ${ }^{2}$. Although this hypothesis is assumed to be valid by many researchers, there is weak scientific evidence in the literature to support this finding ${ }^{3-7}$. Conversely, clinical studies have suggested that diabetics may have good or even excellent functional recovery following decompression of the peripheral nerves in the upper and lower limbs ${ }^{8}$. Similarly, experimental trials have also suggested that good function can be achieved following decompression of nerves in diabetic rats with chronic compression syndromes ${ }^{9}$.

Peripheral neuropathy is one complication frequently observed in diabetic patients ${ }^{10}$. The consequences of peripheral neuropathy have been recognized for some time, and the treatment of one such consequence, plantar ulcers, is critical in preserving the lower limbs. However, the encouraging results from several studies, which have shown good nerve regeneration in diabetic patients, predict that there may have been a change in the natural progression of the disease ${ }^{8,9}$.

The objective of this study was to evaluate nerve regeneration in an experimental model of diabetic rats using a variety of assessments including functional assessments, such as the walking test and electrophysiological examinations; the degree of muscle atrophy; and histomorphometric evaluations of the nerves.

\section{Methods}

The study was approved by the Ethics Committee for the Review of Research Projects at the Clinics Hospital, School of Medicine, University of Sao Paulo. The use of laboratory animals followed the Council for International Organization of Medical Sciences (CIOMS) ethical code for animal experimentation.

Forty 10-week-old male Wistar rats, weighing between 200 and 300 grams, were used. DM was induced in half of the animals, Diabetes Group (D), by intraperitoneal injection of streptozotocin (STZ) solubilized in a citrate buffer solution $(\mathrm{pH}$
4.0) at a dose of $60 \mathrm{mg} / \mathrm{kg}^{11}$. In the other half, Control Group (C), animals were injected with the same amount and dosage of the citrate buffer solution.

\section{Surgical procedures}

After an 8-week period, the animals were placed under general anesthesia with an intraperitoneal injection of pentobarbital sodium $(30 \mathrm{mg} / \mathrm{kg})$ and underwent surgery to expose the sciatic nerve, which was magnified using a microscope.

Each group was further divided into two subgroups of ten rats according to the surgical procedure performed, primary neurorrhaphy $(\mathrm{N})$ or nerve graft $(\mathrm{G})$.

In the neurorrhaphy subgroups (Control - $\mathrm{CN}$ and Diabetes - DN), neurotomy was performed $1 \mathrm{~cm}$ from the bifurcation of the sciatic nerve; primary end-to-end neurorrhaphy was then performed with four separated epineural sutures using a 10/0 monofilament nylon thread.

In the nerve graft subgroups (Control - CG and Diabetes - DG), a segment of $1 \mathrm{~cm}$ of the sciatic nerve was removed and sutured back orthotopically as a graft to repair the respective defect.

At the end of the surgery, we applied a chlorhexidinebased spray-on medication (Merthiolate ${ }^{\square}$ ) directly to the operated limb to avoid self-destruction and mutilation.

The animals' glucose levels were measured prior to DM induction, prior to surgery and at 30 and 60 days postoperatively.

The rats were weighed at different times to evaluate possible changes in baseline weight.

The walking test, which allows for the calculation of the sciatic functional index (SFI) via specific measurements of the rat's footprint ${ }^{12-16}$, was used for functional assessment of sciatic nerve regeneration. The test was performed preoperatively to calculate the baseline value for the SFI and then again at 20, 40 and 60 days postoperatively.

Electrophysiological test was performed at room temperature (approximately $25^{\circ} \mathrm{C}$ ) during surgical re-exploration of the sciatic nerves 60 days postoperatively. The equipment used was NIM (Nerve Integrity Monitoring System, Richards ${ }^{\circledR}$ ) with monopolar stimulation, which triggered an electrical stimulation of $0.30 \mathrm{~mA}$ (milliamp) and a constant latency of $2.0 \mathrm{~ms}$ (milliseconds) directly on the sciatic nerve. The evoked action potential, given in $\mu \mathrm{V}$ (microvolts), was captured by needle electrodes at the center of the triceps surae muscle, and the amplitudes were compared among the subgroups.

The animals were sacrificed by a lethal dose of anesthetic 
administered intraperitoneally. The triceps surae muscles from the operated limb and non-operated limb of each rat were then harvested and weighed on an analytical balance. The weight index (operated limb / non-operated limb) was calculated from these values, which could then be used to compare the degree of muscle atrophy between the subgroups, as described by Siemionow et $a l .^{17}$

Distal to the surgical site, sciatic nerve fragments were collected and fixed by immersion in a 3\% glutaraldehyde solution. Next the segments were gradually dehydrated, first in increasing concentrations of ethyl alcohol $\left(70^{\circ}, 80^{\circ}, 90^{\circ}, 95^{\circ}\right.$ and absolute $1,2,3,4)$, then in a $50 \%$ absolute ethyl alcohol and 50\% xylol mixture. Finally, the sections were cleared in xylol 1, 2, 3, and embedded in paraffin. The embedded material was cut into 2 $\mu \mathrm{m}$ thick cross sections using a microtome and stained by the Hematoxylin and Eosin method and Masson's Trichrome method for hystomorphometric analysis.

Six cross-sections were cut from each nerve to facilitate selection of a section with minimal histological artifacts. The crosssections were collected $0.5 \mathrm{~cm}$ distally from the neurorrhaphy site and the distal suture of the nerve graft.

Image-Pro Plus software, version 4.5 (media cybernetics $^{\circledR}$ ), was used to analyze axonal density, obtained by counting the number of regenerated axons per square micrometer, and the cross-sectional area of the nerve fibers and axons, calculated from measurements of the minimum diameters of the respective structures. The Schwann cell area was obtained by subtracting the area of the axon from the area of the nerve fiber.

The results of the study are expressed as the mean and standard deviation. To compare subgroups, which were defined by the presence of diabetes or the graft, analysis of variance (ANOVA) was used for comparisons of individual factors, and the LSD test (Fisher's least significant difference) was used for multiple comparisons. Student's t-test was used for paired samples to compare the two assessment periods within each subgroup. Repeated measures ANOVA was performed for comparisons involving more than two time periods. The Shapiro-Wilks test was performed to evaluate the normality of the variables. $p<0.05$ were considered statistically significant.

\section{Results}

Three of the rats died: 1 in the $\mathrm{CN}$ subgroup at 15 days postoperatively, 1 in the DN and 1 in the DG subgroups after DM was induced. For the remaining 37 animals, all of the assessments were performed.

\section{Glucose levels and body weights}

The glucose level and body weight values are shown in Tables 1 and 2, respectively.

All animals (D and C) presented similar glucose levels immediately prior to induction of DM or injection with buffer solution. However, glucose levels in the rats of the diabetes group (DN and DG) were significantly higher than those recorded in the rats of the control group (CN and $\mathrm{CG}$ ) preoperatively (eight weeks after induction of DM or injection with buffer) and at 30 and 60 days postoperatively. There were no significant differences observed in glucose levels of the animals of the same group, control or diabetes.

Preoperatively, the body weights of the animals were similar and there was no statistically significant difference between the groups and subgroups.

At 15, 30 and 60 days postoperatively, assessments showed that the weight of the animals of the control group was significantly higher than that of the diabetes group. No significant differences were observed among the animals of the same group, control or diabetes.

\section{Walking test}

In two rats, one in the CG subgroup and one in the DG subgroup, self-mutilation was observed on the operated limb of the animal, preventing the rat from performing the walking test. Table 3 shows the SFI values for the evaluations at the different time points; the values are expressed as a percentage of the loss of normal function (values of $0 \pm 10$ are considered normal). Figure 1 illustrates the evolution of the index for each subgroup at the different time points. 
Salles Jr GS et al.

TABLE 1 - Mean and standard deviation of the blood glucose level (mg/dl) measurement for the subgroups at different assessment periods. P-values compare the four subgroups.

\begin{tabular}{ccccc}
\hline SUBGROUP & $\begin{array}{c}\text { pre-DM induction or } \\
\text { inoculation buffer }\end{array}$ & $\begin{array}{c}\text { pre-operative (8 weeks } \\
\text { after induction or } \\
\text { inoculation) }\end{array}$ & 30 days PO & 60 days PO \\
\hline CN & $94.6(\mathrm{a}) \pm 5.7$ & $92.4(\mathrm{a}) \pm 4.8$ & $92.5(\mathrm{a}) \pm 3.6$ & $92.2(\mathrm{a}) \pm 4.2$ \\
\hline $\mathrm{CG}$ & $93.5(\mathrm{a}) \pm 7.4$ & $91.7(\mathrm{a}) \pm 6.2$ & $91.8(\mathrm{a}) \pm 3.2$ & $92.5(\mathrm{a}) \pm 3.7$ \\
\hline $\mathrm{DN}$ & $94.9(\mathrm{a}) \pm 4.1$ & $427.7(\mathrm{~b}) \pm 107.8$ & $334.4(\mathrm{~b}) \pm 67.6$ & $327.8(\mathrm{~b}) \pm 60.8$ \\
\hline DG & $93.6(\mathrm{a}) \pm 5.6$ & $439.2(\mathrm{~b}) \pm 61.4$ & $326.5(\mathrm{~b}) \pm 36.8$ & $321.5(\mathrm{~b}) \pm 48.4$ \\
\hline p-value & 0.931 & $<0.001$ & $<0.001$ & $<0.001$ \\
\hline
\end{tabular}

Different letters indicate statistically significant differences.

TABLE 2 - Mean and standard deviation of the body weight for each group at the different assessment periods. P-values compare the four subgroups.

\begin{tabular}{ccccc}
\hline SUBGROUP & pre-operation & 15 days PO & 30 days PO & 60 days PO \\
\hline CN & $277.4(\mathrm{a}) \pm 10.5$ & $260.3(\mathrm{a}) \pm 8.8$ & $304.8(\mathrm{a}) \pm 19.0$ & $352.7(\mathrm{a}) \pm 22.6$ \\
\hline CG & $279.3(\mathrm{a}) \pm 17.8$ & $265.4(\mathrm{a}) \pm 19.7$ & $309.7(\mathrm{a}) \pm 16.9$ & $355.6(\mathrm{a}) \pm 22.2$ \\
\hline DN & $281.2(\mathrm{a}) \pm 12.3$ & $225.1(\mathrm{~b}) \pm 19.9$ & $257.8(\mathrm{~b}) \pm 19.5$ & $301.9(\mathrm{~b}) \pm 14.9$ \\
\hline DG & $279.1(\mathrm{a}) \pm 11.9$ & $223.0(\mathrm{~b}) \pm 13.7$ & $258.4(\mathrm{~b}) \pm 19.1$ & $305.1(\mathrm{~b}) \pm 14.2$ \\
\hline p-value & 0.939 & $<0.001$ & $<0.001$ & $<0.001$ \\
\hline
\end{tabular}

Different letters indicate statistically significant differences.

TABLE 3 - Mean and standard deviation of the Sciatic Function Index (SFI) for the subgroups at the different time points. P-values compare the four subgroups.

\begin{tabular}{ccccc} 
SUBGROUP & pre-operation & $\mathbf{2 0}$ days PO & $\mathbf{4 0}$ days PO & $\mathbf{6 0}$ days PO \\
\hline CN & $-6.5(\mathrm{a}) \pm 1.1$ & $-25.9(\mathrm{a}) \pm 4.0$ & $-10.7(\mathrm{a}) \pm 1.6$ & $-12.7(\mathrm{a}) \pm 1.0$ \\
\hline CG & $-6.0(\mathrm{a}) \pm 0.8$ & $-36.7(\mathrm{~b}) \pm 4.1$ & $-12.6(\mathrm{~b}) \pm 2.1$ & $-12.9(\mathrm{a}) \pm 1.6$ \\
\hline DN & $-6.7(\mathrm{a}) \pm 1.1$ & $-67.8(\mathrm{c}) \pm 4.6$ & $-15.3(\mathrm{c}) \pm 1.6$ & $-12.9(\mathrm{a}) \pm 1.1$ \\
\hline DG & $-6.0(\mathrm{a}) \pm 0.5$ & $-79.3(\mathrm{~d}) \pm 7.2$ & $-14.8(\mathrm{c}) \pm 1.8$ & $-14.4(\mathrm{~b}) \pm 0.9$ \\
\hline p-value & 0.224 & $<0.001$ & $<0.001$ & 0.007
\end{tabular}

Different letters indicate statistically significant differences. 


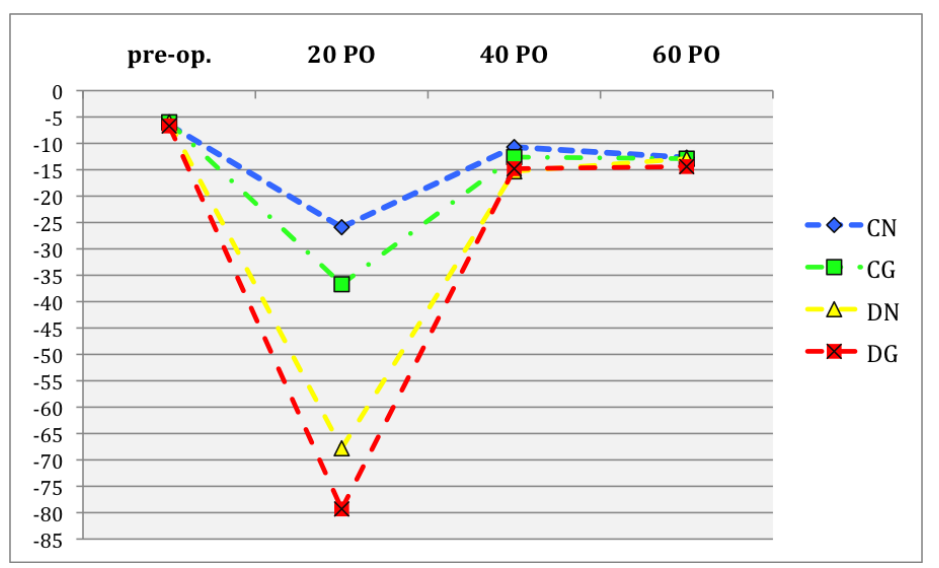

FIGURE 1 - SFI evolution (\% loss of normal function).

The preoperative SFIs were similar between the groups and subgroups, with no statistically significant difference observed.

Evaluation 20 days post surgery showed a statistically significant difference between the diabetes and control groups. Upon comparing the SFI value in each subgroup, control or diabetes, it was observed that the indexes of the animals submitted to neurorrhaphy was closer to normal than the animals submitted to nerve graft, with statistically significant differences.

The values at 40 days remained essentially unchanged, with the exception that within the group of diabetic animals there was no statistically significant difference between subgroups DN and DG.

At 60 days, the SFI values in subgroups CN, CG and DN were statistically better than those recorded in subgroup DG.

\section{Electrophysiological test}

Although the evoked potential amplitudes reached their highest levels in the $\mathrm{CN}$ subgroup $(350 \mu \mathrm{V} \pm 87.9)$, the values were statistically similar ( $>>0.05)$ to those obtained in the other subgroups: $\mathrm{CG}(285.2 \mu \mathrm{V} \pm 88.8), \mathrm{DN}(299.2 \mu \mathrm{V} \pm 67.5)$ and $\mathrm{DG}$ $(311.0 \mu \mathrm{V} \pm 72.5)$.

\section{Index of triceps surae muscle weights}

The mean index for the CN subgroup $(0.78 \mathrm{~g} \pm 0.2)$ was significantly higher $(\mathrm{p}<0.001)$ than the mean index for the $\mathrm{CG}$ (0.59 $\mathrm{g} \pm 0.2), \mathrm{DN}(0.50 \mathrm{~g} \pm 0.2)$ and $\mathrm{DG}(0.50 \mathrm{~g} \pm 0.2)$ subgroups. None of these last three subgroups were significantly different from each other.

\section{Histomorphometric analysis}

The axonal densities and cross-sectional areas of the nerve fibers, axons and Schwann cells were evaluated. These measurements were not statistically different between the diabetic and control subgroups. Figures 2 to 5 illustrates these results, including the mean, standard deviation and p-values for the comparisons among the four subgroups.

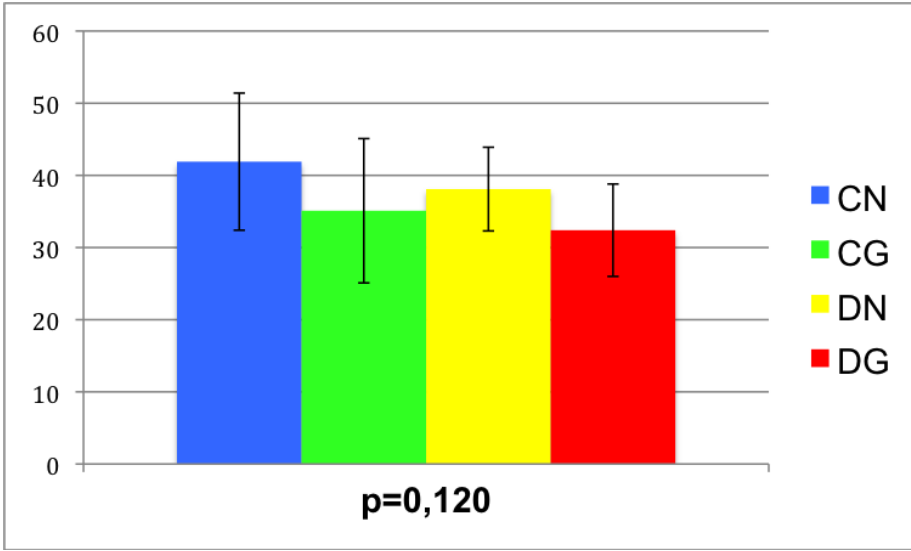

FIGURE 2 - Axonal density (axons/11869.33 $\mu 2$ ).

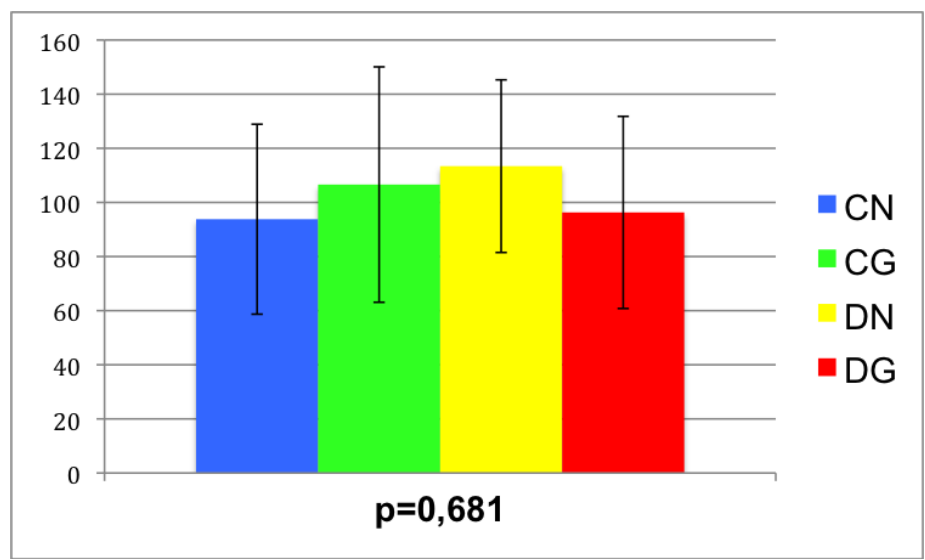

FIGURE 3 - Nerve fiber area $(\mu 2)$.

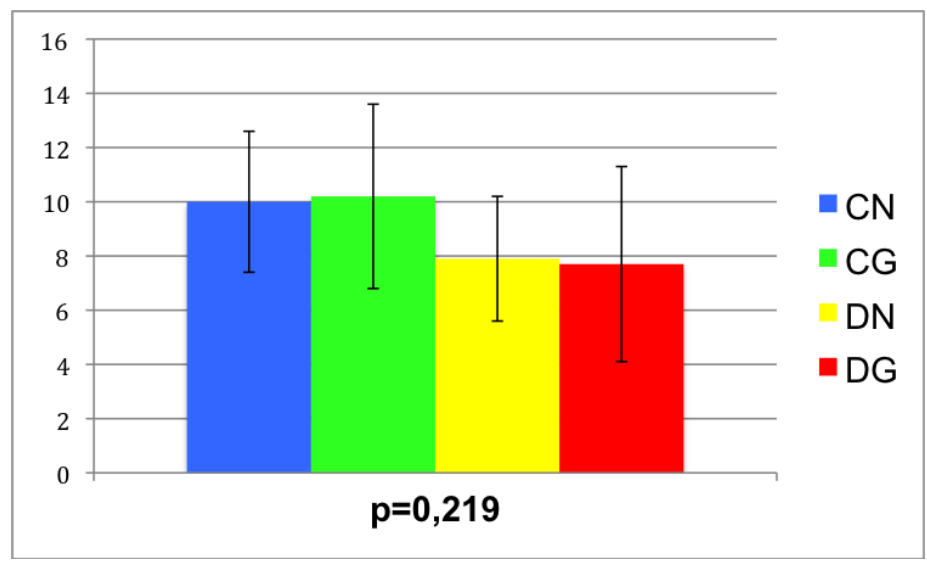

FIGURE 4 - Axon area $(\mu 2)$. 


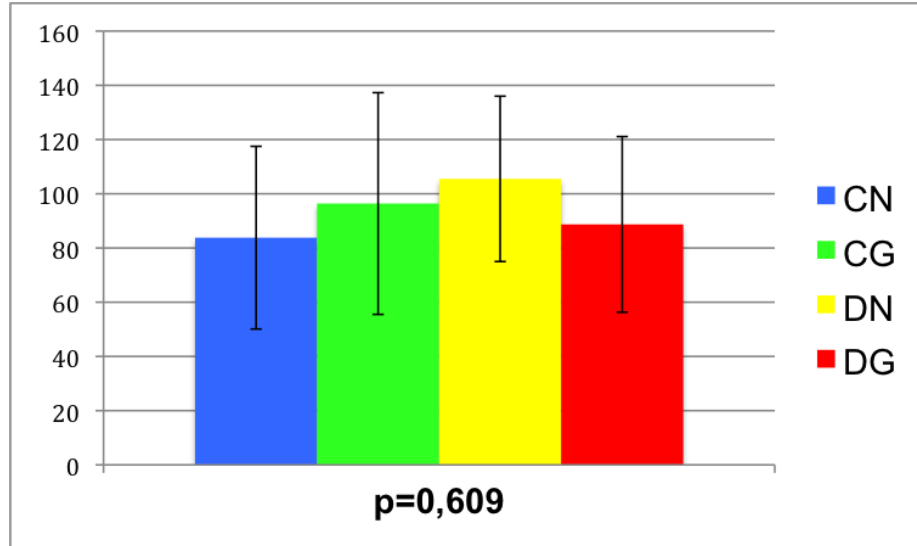

FIGURE 5 - Schwann cell area $(\mu 2)$.

Axonal density was higher in the subgroups with neurorrhaphy than the subgroups with graft, in both the control group and the diabetic group. However, no statistically significant differences were found comparing $\mathrm{CN}$ with $\mathrm{DN}$ and comparing CG with DG (Figure 6).

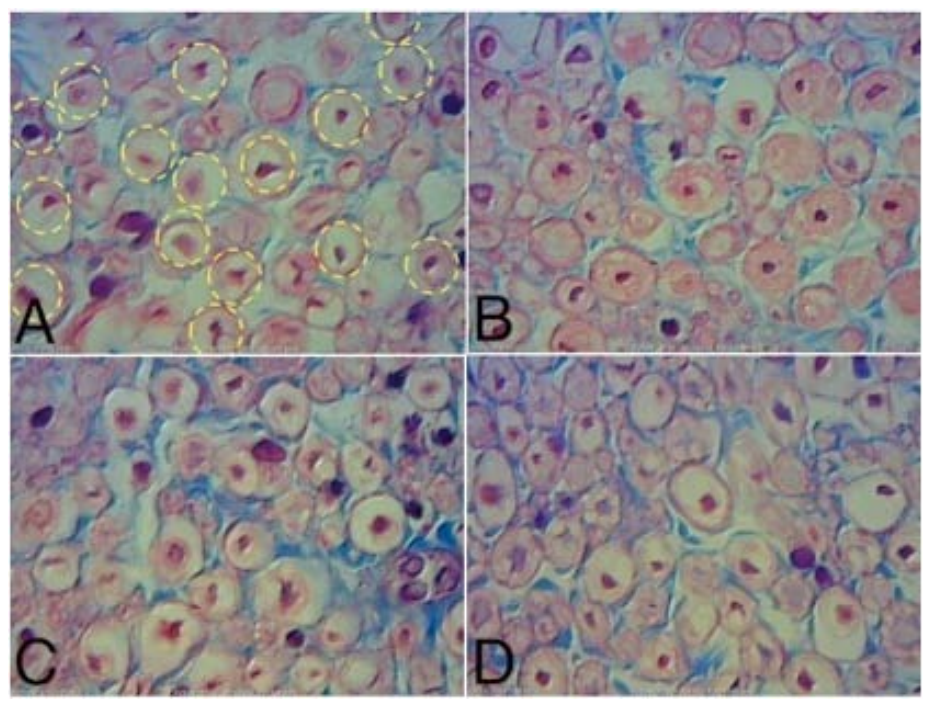

FIGURE 6 - Photomicrographs showing axonal density of sciatic nerve segments of the axons (highlighted in yellow dotted circles in panel A) in the neural stroma: CN (A), CG (B), DN (C) and DG (D). Masson's trichrome staining. Optical microscopy at x400 magnification.

The mean cross-sectional area of the nerve fibers was largest in subgroup DN, followed by subgroups CG, DG and CN, respectively (Figure 7).

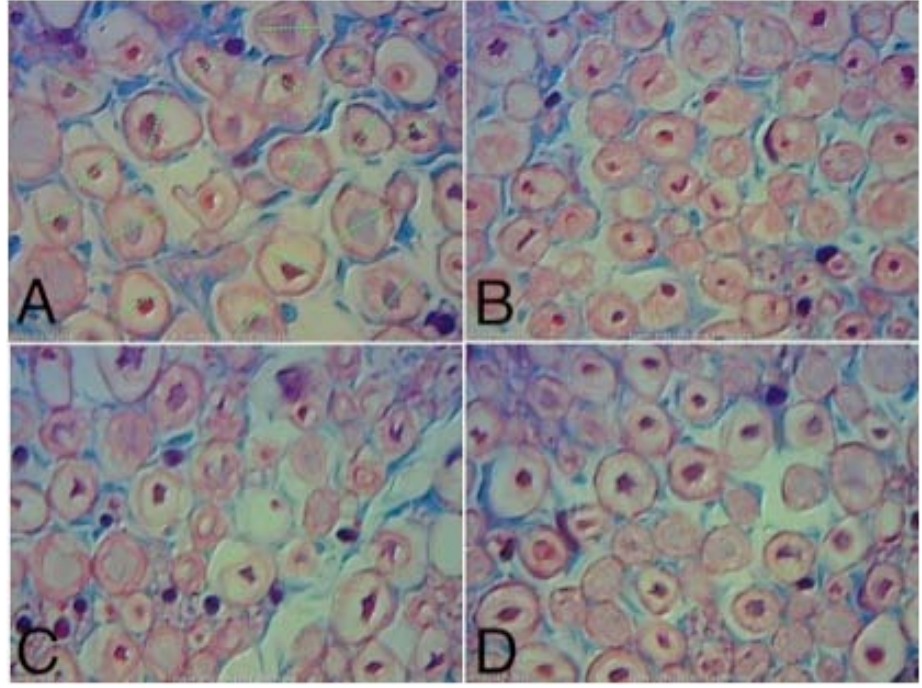

FIGURE 7 - Photomicrographs of cross-sections of the distal segment of the sciatic nerve (green dotted lines in panel A correspond to the smallest diameter of the transverse sections of the nerve fibers): CN (A), CG (B), DN (C) and DG (D). Masson's trichrome staining. Optical microscopy at x400 magnification.

With respect to the mean cross-sectional area of the axons, the highest values were observed in subgroups $\mathrm{CG}$ and $\mathrm{CN}$, followed by subgroups DN and DG (Figure 8).

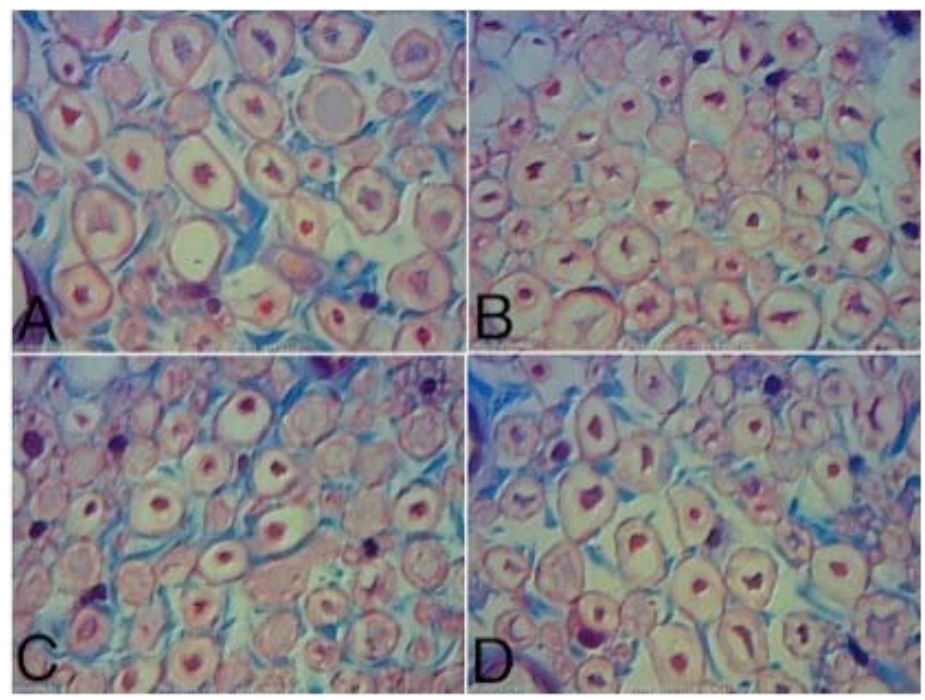

FIGURE 8 - Photomicrographs of cross-sections of the distal segment of the sciatic nerve (red dotted lines in panel A correspond to the smallest diameter of the transverse sections of the axons): $\mathrm{CN}(\mathbf{A}), \mathrm{CG}(\mathbf{B}), \mathrm{DN}(\mathbf{C})$ and DG (D). Masson's trichrome staining. Optical microscopy at x400 magnification.

The measurements of the mean cross-sectional area of the Schwann cells presented a pattern identical to the those of the mean cross-sectional area of the nerve fibers, which were largest in subgroup DN, followed by CG, DG and CN. 


\section{Discussion}

Several experimental and clinical studies suggest a decrease in nerve regeneration in the presence of diabetes ${ }^{3-7}$. In order to obtain a more detailed and comprehensive analysis, functional, electrophysiological, anatomical and histological parameters were used in this study.

After eight weeks, the rats given a single intraperitoneal streptozotocin injection presented significantly higher glucose levels than the rats given a buffer injection, characterizing them diabetics and non-diabetics, respectively. At this point, the diabetic animals had significantly lower weight than the nondiabetic control animals, which is consistent with the results of other studies ${ }^{18,19}$

The walking test evaluates the gait of a rat and is a reliable method for functionally evaluating nerve regeneration ${ }^{16,20,21}$. Among the rats submitted to sciatic nerve repair with neurorrhaphy, the diabetic rats had significantly lower SFI values than the non-diabetic rats in evaluations performed at 20 and 40 days postoperatively. However, in the last functional evaluation performed at 60 days postoperatively, the values were statistically similar. The apparent delay in the speed of nerve regeneration in the diabetic rats did not definitively affect the final functional recovery.

There is a lack of consensus in the literature on the followup period necessary to effectively analyze nerve regeneration. Knowing that six weeks should be a sufficient period to allow regenerating proximal axons to reach the distal stump of the sciatic nerve $^{22,23}$, we chose a slightly longer time period, 60 days. Babovic et al. ${ }^{18}$ performed their final evaluations at 6 months and found no differences in the walking test between the diabetic and control rats with a graft.

Among the animal submitted to sciatic nerve repair with nerve graft, the SFI values for the diabetic rats remained significantly lower than the non-diabetic rats in the evaluations performed up to 60 days postoperatively. Although the results suggest definitive functional loss in the diabetic rats, we cannot be sure that the regeneration process was completed by the 60 -day follow-up.

The walking-track analysis however, failed to demonstrate differences between neurorrhaphy and nerve grafting in diabetic rats at 40 and 60 days postoperatively. It may not be sensitive enough to detect small functional changes which is consistent with the observations made by Robilard-Goheen et $a l .^{24}$.

Regarding the weight variation in the animals during the postoperative walking test assessment, we selected age- matched animals, unlike other studies that only paired the animals according to weight ${ }^{9,18}$. The SFI is calculated from three footprint measurements of the rat $^{25}$. Because the animal's body weight is one variable that would apply equally to all three of these measurements, there would be a proportional relationship, and the result would be the same regardless of weight. Pairing animals only by weight while their ages are different may constitute a bias because the aging process affects the organ systems.

The electrophysiological test complemented the functional tests by providing an estimate of the number of functioning axons in the nerve. Because the intensity of electrical stimulation can vary from one study to another, it is difficult to compare isolated amplitude values among studies; furthermore, the intensity of the stimulus determines the amplitude achieved. The results of this study showed that there were no significant differences between the subgroups, indicating that at 60 days postsurgery, the diabetic rats had amplitudes that were similar to those of the controls, which is consistent with the results reported by Kennedy and Zochodne ${ }^{26}$. According to these authors, there may be a slower regeneration rate in diabetic animals; however, our study indicates that the process occurs at a rate similar to that in the control animals.

The degree of muscle atrophy was calculated by using a ratio of muscle weights ${ }^{17}$ between the operated and non-operated sides in an attempt to compensate for the significant variation in body weight among the rats. The mean index was significantly higher in the $\mathrm{CN}$ subgroup than in the other three subgroups, likely indicating that reinnervation of the triceps surae muscle occurred in these animals before it took place in the animals of the other subgroups. Because muscle atrophy on one side is usually accompanied by muscle hypertrophy on the contralateral side, an increase of this index during recovery is expected to be a reliable parameter of the degree of muscle reinnervation. However, the results do not allow us to assure that muscle reinnervation was incomplete at 60 days post-surgery or if it was definitively compromised.

In the histomorphometry analysis, the cross-sectional areas of the structures were calculated by measuring their smallest diameter because it does not change with the slope of the histological section ${ }^{27}$. De Sá et al. ${ }^{28}$ reported that measuring the minimum diameter of the axonal fibers is the most reliable parameter for nerve regeneration. The present study showed that the axonal density was not statistically different between groups and subgroups.

Kennedy and Zochodne ${ }^{26}$ found a smaller axonal diameter and a decrease of myelin sheaths after crush and 
transactional injuries in diabetic rats compared to the non-diabetic ones. These differences were not fully reproduced by others ${ }^{29,30}$. According to Yasihashi et al. ${ }^{31}$, the correlation between the density of remyelinated fibers and functional changes is debatable. In this study, non-diabetic rats submitted to neurorrhaphy showed smaller values of areas of cross-sections of the nerve fibers, areas of axons and areas of Schwann cell and also, demonstrated the best functional results (higher SFI values).

Thomas $^{32}$ and De Medinaceli ${ }^{33}$ reported that the accuracy with which regenerated axons reestablish connections with the target organ is more important than the total number of fibers. This study reinforces the statement that histological characteristics can provide a reliable picture of the trophic conditions of the regenerated nerve, but they do not directly correlate with the degree of functional recovery.

The promising results of this study, which demonstrated good regeneration in diabetic rats via various forms of assessment, offer a perspective for studies of nerve decompression in patients with diabetic foot ulcers refractory to conventional treatment because this study demonstrates that nerve regeneration can occur, albeit more slowly and with difficulty. Therefore, there is a chance to change the natural history of these patients, who often require lower limb amputation and have lower long-term life expectancies.

\section{Conclusion}

The nerve regeneration in diabetic rats was slower but was not significantly jeopardized at 60 days post-surgery.

\section{References}

1. Danaei G, Finucane MM, Lu Y, Singh GM, Cowan MJ, Paciorek CJ, Lin JK, Farzadfar F, Khang YH, Stevens GA, Rao M, Ali MK, Riley LM, Robinson CA, Ezzati M. National, regional, and global trends in fasting plasma glucose and diabetes prevalence since 1980: systematic analysis of health examination surveys and epidemiological studies with 370 country-years and 2.7 million participants. Lancet. 2011;378(9785):31-40.

2. Kennedy JM,Zochodne DW. Influence of experimental diabetes on the microcirculation of injured peripheral nerve: functional and morphological aspects. Diabetes. 2002;51(7):2233-40.

3. Eliasson SG. Regeneration process in experimental diabetic neuropathies. Trans Am Neurol Assoc. 1965;90:35-7.

4. Sharma AK, Thomas PK. Peripheral nerve regeneration in experimental diabetes. J Neurol Sci. 1975;24:417-24.

5. Bisby MA. Axonal transport of labeled proteins and regeneration rate in nerves of streptozotocin-diabetic rats. Exp Neurol. 1980;69:7484.

6. Sharma AK, Bajada S, Thomas PK. Influence of streptozotocininduced diabetes on myelinated nerve fibre maturation and on body growth in the rat. Acta Neuropathol. 1981;53(4):257-65.

7. Longo FM, Powell HC, Lebeau J, Guerrero MR, Heckman H,
Myers RR. Delayed nerve regeneration in streptozotocin diabetic rats. Muscle Nerve. 1986;9:385-93.

8. Dellon AL. Treatment of symptoms of diabetic neuropathy by surgical decompressions of multiple peripheral nerves. Plast Reconstr Surg. 1992;89:869-97.

9. Dellon AL, Dellon ES, Seiler WA. Effect of tarsal tunnel decompression in the streptozotocin-induced diabetic rat. Microsurgery. 1994;5:265-8.

10. Powell HC, Rosoff J, Myers RR. Microangiopathy in human diabetic neuropathy. Acta Neuropathol. 1985;68:295-305.

11. Boner W, Trent DS, Honey RN, Weir GS. Response of neonatal islets to streptozotocine - limited cell regeneration and hyperglycemia. Diabetes. 1981;30(1):64-9.

12. Brown CJ, Mackinnon SE, Evans PJ, Bain JR, Makino AP, Hunter DA, Hare GMT. Self-evaluation of walking-track measurement using a sciatic function index. Microsurgery. 1989;10:226-35.

13. Brown CJ, Evans PJ, Mackinnon SE, Bain JR, Makino AP, Hunter DA, Hare GMT. Inter- and intraobserver reliability of walking-track analysis used to assess sciatic nerve function in rats. Microsurgery. 1991;12:76-9.

14. Dash H, Kononov A, Prayson RA, Petras S, Browne EZ. Evaluation of nerve recovery from minimal duration crush injury. Ann Plast Surg. 1996;37(5):526-31.

15. Hare GMT, Evans PJ, Mackinnon SE, Best TJ, Bain JR, Szalai JP, Hunter DA. Walking track analysis: a long-term assessment of peripheral nerve recovery. Plast Reconst Surg. 1992;89(2):251-8.

16. Hare GMT, Evans PJ, Mackinnon SE, Best TJ, Midha R, Szalai JP, Hunter DA. Walking track analysis: utilization of individual footprint parameters. Ann Plast Surg. 1993;30(2):147-53.

17. Siemionow M, Sari A, Demir Y. Effect of early nerve release on the progression of neuropathy in diabetic rats. Ann Plast Surg. 2007;59:102-8.

18. Babovic S, Ress AM, Dellon AL, Angel MF, Im MJ, Manson PN. Nerve regeneration in diabetic rats. Microsurgery. 1998;18:9-11.

19. Dellon AL, Mackinnon SE, Seiler IV WA. Susceptibility of the diabetic nerve to chronic compression. Ann Plast Surg. 1988;20(2):117-9.

20. Dellon ES, Dellon AL. Functional assessment of neurologic impairment: Track analysis in diabetic and compression neuropathies. Plast Reconstr Surg. 1991;88:686-94.

21. Mackinnon SE, Dellon AL. Two-point discrimination tester. J Hand Surg Am. 1985;10:906-7.

22. Stancic MF, Micovic V, Potocnjak M, Draganic P, Sasso A, Mackinnon SE. The value of an operating microscope in peripheral nerve repair. Int Orthop. 1988;22:107-10.

23. Fansa H, Keilhoff G, Altmann S, Plogmeier K, Wolf G, Schneider W. The effect of immunosuppressant FK506 on peripheral nerve regeneration following nerve grafting. J Hand Surg. 1999;24:38-42.

24. Goheen-Robilard B, Myckatyn TM, Mackinnon SE, Hunter DA. End-to-side neurorrhaphy and lateral axonal sprouting in a long graft rat model. Laryngoscope. 2002;112:899-905.

25. Bain JR, Mackinnon SE, Hunter DA. Functional evaluation of complete sciatic, peroneal and posterior tibial nerve lesions in the rat. Plast Reconstr Surg. 1989;83:129-36.

26. Kennedy JM, Zochodne DW. The regenerative deficit of peripheral nerves in experimental diabetes: its extent, timing and possible mechanisms. Brain. 2000;123:2118-29.

27. Romão AM, Viterbo F, Stipp EJ, Garbino JA, Rodrigues JA. Muscle electro stimulation of the cranial tibial muscle after crushing of the common fibular nerve: neurophysiologic and morphometric study in rats. Rev Bras Ortop. 2007;42(3):41-6.

28. De Sá JMR, Mazzer N, Barbieri CH, Barreira AA. The end-to-side 
peripheral nerve repair functional and morphometric study using the peroneal nerve of rats. J Neurosc Meth. 2004;136(1):45-53.

29. Jakobsen J, Lundbaeck K. Neuropathy in experimental diabetes: an animal model. Br Med J. 1976;2:178-279.

30. Machado JLM, Macedo AR, Silva MD, Spadella CT, Montenegro MRG. Caracterização de um modelo experimental de neuropatia em ratos diabéticos induzido pela aloxana. Acta Cir Bras. 2000;15(2):132-42.

31. Yasihashi S, Nishikira M, Baba M. Morphometrical analysis of the peripheral nerve lesions in experimental diabetes rats. J Exp Med. 1979;129:139-99.

32. Thomas PK. The cellular response to nerve injury. The effect of repeated crush injuries. J Anat. 1970;106:463-70.

33. De Medinaceli L. Interpreting nerve morphometry data after experimental traumatic lesions. J Neurosci Methods. 1995;58:2937.

\section{Correspondence:}

Guataçara Schenfelder Salles Jr

Rua Eduardo Sprada, 1872/casa 38

81210-370 Curitiba - PR Brasil

Tel.: (55 41)9188-1978

guatajr@yahoo.com.br

Received: March 15, 2013

Review: May 17, 2013

Accepted: June 18, 2013

Conflict of interest: none

Financial source: none

${ }^{1}$ Research performed at Laboratory of Experimental Microsurgery,

Division of Plastic Surgery, Department of Surgery, School of Medicine, University of Sao Paulo (FMUSP), Brazil. Part of PhD degree thesis, Postgraduate Program in Clinical Surgery. Tutor: José Carlos Marques de Faria. 